\title{
CHORD PROPERTIES OF DIGITAL STRAIGHT LINE SEGMENTS
}

\author{
SHIVA SAMIEINIA
}

\begin{abstract}
We exhibit the structure of digital straight line segments in the 8-connected plane and in the Khalimsky plane by considering vertical distances and unions of two segments.
\end{abstract}

\section{Introduction}

In the field of digital geometry one of the themes which has been studied extensively is digital straight lines. Malon̆ and Freeman [12] and Freeman [3] introduced the chain code as a technique for representing 8-connected arcs and lines. The most important problem related to straightness is how to recognize the sets of pixels or codes representing a digital straight line. Rosenfeld [16] characterized straightness by the chord property and found two fundamental properties of run lengths in a digital line. He stated that the digitized line can only contain runs of two different lengths and their lengths must be consecutive integers. To decompose the digital curves, Smeulders and Dorst [17] worked with runs as the sequences of successive elements in Freeman's code with the same values, and go to the higher order by classifying runs with the same number of elements. They showed there are two different values for the runs of nonfinal orders. Uscka-Wehlou [18] studied digital lines with irrational slopes by considering runs.

Hung and Kasvand [4] gave a necessary and sufficient condition for a digital arc to have the chord property. This condition made the chord property easier to check. Kim [7] characterized it by convexity, and showed that a digital straight line segment is a digital arc which is digitally convex. Reveillès [15] introduced the concept of naive digital line by double Diophantine inequalities. As a generalization of this definition he defined a naive digital hyperplane. Kiselman [8] generalized Reveillès' definition of a digital hyperplane by allowing more freely strict and non-strict inequalities. He represented a digital hyperplane as a graph of a function which is both convex and concave.

Received 29 February 2008. 
Bruckstein [2] presented some transformations on sequences composed of two symbols, 0 and 1 . These transformations can be described by matrices which form a well-known group called $\mathrm{GL}(2, \mathrm{Z})$. The main result in his paper is that the image of the chain code under one of these transformations represents a digital straight line segment if and only if the original sequence is the chain code of a digital straight line segment. Similar transformations have been used by Jamet and Toutant [5] in the case of three dimensions. Arnoux et al. [1] have worked with three dimensional digital hyperplanes. Klette and Rosenfeld [11] presented a review on the concepts of digital lines. They also published a book [10], which provides citations of important researches of that field.

In the present paper we deal with grid points in the 8-connected plane as well as the plane equipped with the Khalimsky topology. Digital straight line segments are special cases of digital arcs. We shall investigate Rosenfeld's digitization and his chord property in Section 1.1. Melin [13] introduced a modified version of the chord property of Rosenfeld. He established necessary and sufficient conditions for straightness in the Khalimsky plane which we mention in Section 1.3.

To investigate straightness the 8-connected and Khalimsky-connected points, in Section 2 we introduce the notion of boomerang by dividing the points into unions of horizontal and diagonal segments. Then we present necessary and sufficient conditions for straightness in both cases using vertical distances for certain points. It puts the straightness in both planes into a new framework and makes investigating straightness easier to check.

In Section 4 we shall go through the properties of boomerangs for digital lines and state necessary conditions for straightness. We conclude also in Lemmas 4.6 and 4.7 some similar results for the cardinality of boomerangs as Smeulders and Dorst [17] found for the runs of nonfinal orders. The conditions obtained in these two lemmas will be shown not to be sufficient for straightness. We shall also establish necessary and sufficient conditions in the 8-connected plane as well as in the Khalimsky plane by transforming the sequences of their chain codes. Using this technique, we transform Khalimsky lines to the 8-connected case. The result of this transformation allows us to escape from topological connectedness and choose the 8-connected techniques to characterize the Khalimsky-connected one.

\subsection{Rosenfeld's digitization of straight lines}

We present here Rosenfeld's digitization of straight lines in the digital plane $Z^{2}$. First we define the set

$$
\begin{aligned}
C(0)=\left\{x ; x_{1}=0 \text { and }-1 / 2<\right. & \left.x_{2} \leq 1 / 2\right\} \\
& \cup\left\{x ; x_{2}=0 \text { and }-1 / 2<x_{1} \leq 1 / 2\right\} .
\end{aligned}
$$


For each $p \in \mathrm{Z}^{2}$, let $C(p)=C(0)+p$, which we shall call the cross with center $p$. Now the Rosenfeld digitization in $\mathrm{R}^{2}$ is:

$$
D_{R}: \mathrm{P}\left(\mathrm{R}^{2}\right) \rightarrow \mathrm{P}\left(\mathrm{Z}^{2}\right), \quad D_{R}(A)=\left\{p \in \mathrm{Z}^{2} ; C(p) \cap A \neq \emptyset\right\} .
$$

This digitization is based on the one-dimensional digitization

$$
\mathrm{R} \ni x \mapsto\lceil x-1 / 2\rceil \in \mathrm{Z} .
$$

The union of all crosses $C(p)$ for $p \in \mathrm{Z}^{2}$ is equal to the set of all grid lines $(R \times Z) \cup(Z \times R)$, so that every straight line has a nonempty digitization. Note that the family of all crosses is disjoint, which implies that the digitization of a point is either empty or a singleton set. In the real plane, the concept of a straight line is well-known: it is a set of the form $\{(1-t) a+t b ; t \in \mathrm{R}\}$, where $a$ and $b$ are two distinct points in the plane. A straight line segment is a connected subset of a straight line (perhaps the whole line).

We shall consider in particular closed segments of finite length and we write them as $\{(1-t) a+t b ; 0 \leq t \leq 1\}$, where $a$ and $b$ are the endpoints. We shall denote this segment by $[a, b]$. Like Rosenfeld, we will consider lines and straight line segments with slope between 0 and $45^{\circ}$ in the 8 -connected case and in the Khalimsky plane.

We shall say that $D$ is a digital straight line segment, and write $D \in \mathrm{DSLS}_{8}$, if and only if there exists a real line segment the Rosenfeld digitization of which is equal to $D$.

Rosenfeld [16] introduced the chord property to characterize digital straight line segments in $Z^{2}$ :

Definition 1.1. A subset $D \subseteq \mathrm{R}^{2}$ is said to have the chord property if for all points $p, q \in D$ the segment $[p, q]$ is contained in $D+B_{<}^{\infty}(0,1)$, the dilation of $D$ by the open unit ball for the $l^{\infty}$ metric.

Rosenfeld's digitization of a subset in the plane $Z^{2}$ is 8-connected, but if we consider it in the Khalimsky plane, it is not necessarily connected for that topology. Also, we do not have the chord property with respect to the $l^{\infty}$ distance for certain Khalimsky-connected sets which are digitizations of straight line segments. Melin [13] solved these problems by suggesting another digitization and modified Rosenfeld's chord property. To explain this, we shall start with the definition of the Khalimsky plane and then continue with Melin's digitization.

\subsection{The Khalimsky topology}

There are several different ways to introduce the Khalimsky topology on the integer line. We present the Khalimsky topology by a topological basis. For 
every even integer $m$, the set $\{m-1, m, m+1\}$ is open, and for every odd integer $n$ the singleton $\{n\}$ is open. A basis is given by

$$
\{\{2 n+1\},\{2 n-1,2 n, 2 n+1\} ; n \in \mathrm{Z}\} .
$$

It follows that even points are closed.

A digital interval $[a, b]_{z}=[a, b] \cap Z$ with the subspace topology is called a Khalimsky interval, and a homeomorphic image of a Khalimsky interval into a topological space is called a Khalimsky arc.

On the digital plane $Z^{2}$, the Khalimsky topology is given by the product topology. A point with both coordinates odd is open. If both coordinates are even, the point is closed. These types of points are called pure. Points with one even and one odd coordinate are neither open nor closed; these are called mixed. Note that the mixed points are only connected to their 4-neighbors, whereas the pure points are connected to all eight neighbors. More information on the Khalimsky plane and the Khalimsky topology can be found in Khalimsky et al. [6], Kiselman [9] and Melin [14].

\subsection{Continuous Khalimsky digitization}

The Rosenfeld digitization in $\mathrm{R}^{2}$ does not work well when $\mathrm{Z}^{2}$ is equipped with the Khalimsky topology. This means that the Rosenfeld digitization of a straight line segment is not in general connected for the Khalimsky topology. Melin [13] introduced a Khalimsky-continuous digitization. This digitization gives us Khalimsky-connected digital straight line segments.

Here we recall his definition and related results. Let

$$
D(0)=\left\{(t, t) \in \mathrm{R}^{2} ;-1 / 2<t \leq 1 / 2\right\} \cup\left\{(t,-t) \in \mathrm{R}^{2} ;-1 / 2<t \leq 1 / 2\right\} .
$$

For each pure point $p \in \mathrm{Z}^{2}$, define $D(p)=D(0)+p$. Note that $D(p)$ is a cross, rotated $45^{\circ}$, with center at $p$, and that $D(p)$ is contained in the Voronoi cell $\left\{x \in \mathrm{R}^{2} ;\|x-p\|_{\infty} \leq 1 / 2\right\}$. This means that a digitization with $D(p)$ as a cross with nucleus $p$ is a Voronoi digitization. We define the pure digitization $D_{P}(A)$ of a subset $A$ of $\mathrm{R}^{2}$ as

$$
D_{P}(A)=\left\{p \in Z^{2} ; p \text { is pure and } D(p) \cap A \neq \emptyset\right\} .
$$

This digitization is the basis for the continuous digitization. Let $L$ be a Khalimsky-connected set of points. The continuous digitization $D(L)$ of $L$ is defined as follows: If $L$ is horizontal or vertical $D(L)=D_{R}(L)$, the Rosenfeld digitization defined in (1). Otherwise define $D_{M}(L)$ as

$$
D_{M}(L)=\left\{p \in \mathrm{Z}^{2} ;\left(p_{1} \pm 1, p_{2}\right) \in D_{P}(L)\right\} \cup\left\{p \in \mathrm{Z}^{2} ;\left(p_{1}, p_{2} \pm 1\right) \in D_{P}(L)\right\}
$$


and let $D(L)=D_{P}(L) \cup D_{M}(L)$. In this digitization we add mixed points $\left(p_{1}, p_{2}\right)$ if the two points $\left(p_{1} \pm 1, p_{2}\right)$ or the two points $\left(p_{1}, p_{2} \pm 1\right)$ belong to the pure digitization. Melin [13] characterized digital straight line segments in the 8-connected and the Khalimsky-connected cases by using a function which he called chord measure.

Definition 1.2. Let $A \in \mathrm{P}_{\text {finite }}\left(\mathrm{Z}^{2}\right)$ be a finite set. Then the chord measure of $A$, denoted by $\xi(A)$, is defined by:

$$
\xi(A)=\max _{p, q \in A} H(A, p, q),
$$

where $H(A, p, q)$ is the distance from the line segment $[p, q]$ to $A$, which is defined by

$$
H: \mathrm{P}_{\text {finite }}\left(\mathrm{Z}^{2}\right) \times \mathrm{Z}^{2} \times \mathrm{Z}^{2} \rightarrow[0,+\infty], \quad H(A, p, q)=\sup _{x \in[p, q]} \min _{m \in A} d(m, x) .
$$

The distance function $H$ is related to the Hausdorff distance between $A$ and $[p, q]$ as two subsets of the metric space $\left(\mathrm{Z}^{2}, d\right)$.

Definition 1.3. Let $A \in \mathrm{P}_{\text {finite }}\left(\mathrm{Z}^{2}\right)$. We say that $A$ has the chord property for the metric $d$ if $\xi(A)<1$.

As to the Rosenfeld digitization, Melin [13] showed that a continuous Khalimsky digitization satisfies the chord property for a certain metric and, conversely, a Khalimsky arc satisfying this chord property is the digitization of a straight line segment. He considered a special metric. Let $\delta^{\infty}$ be the metric on $R^{2}$ defined by

$$
\delta^{\infty}(x, y)=\max \left(\frac{1}{2}\left|x_{1}-y_{1}\right|,\left|x_{2}-y_{2}\right|\right) ;
$$

it is the $l^{\infty}$-metric rescaled in the first coordinate. For each positive $\alpha$, we may define a metric $\delta_{\alpha}^{\infty}(x, y)=\max \left(\alpha\left|x_{1}-y_{1}\right|,\left|x_{2}-y_{2}\right|\right)$, but Melin [13] showed by examples that the choice $\alpha=\frac{1}{2}$ is suitable.

We shall call $D$ a digital straight line segment in the Khalimsky plane, and write $D \in \mathrm{DSLS}_{\mathrm{Kh}}$ if and only if there exists a real straight line segment whose Khalimsky digitization is equal to $D$. Melin [13] proved two theorems that characterize $\mathrm{DSLS}$ Kh.

Theorem 1.4 (Melin [13]: Theorem 6.3). The continuous Khalimsky digitization of a straight line segment is a Khalimsky arc (possibly empty) having the chord property for the $\delta^{\infty}$-metric (when the slope is between $0^{\circ}$ and $45^{\circ}$ ) or the metric $\delta(x, y)=\delta\left(\left(x_{2}, x_{1}\right),\left(y_{2}, y_{1}\right)\right)$ (for lines with slope between $45^{\circ}$ and $90^{\circ}$ ). 
Theorem 1.5 (Melin [13]: Theorem 6.4). Suppose that a Khalimsky arc

$$
D=\{(x, f(x)) ; x \in I\} \subseteq Z^{2}
$$

is the graph of a monotone, continuous function $f$, and that $D$ has pure endpoints. If $D$ has the chord property for the $\delta^{\infty}$-metric, then $D$ is the Khalimskycontinuous digitization of a straight line segment.

Remark 1.6. Melin [13] defined another way to distinguish DSLS $\mathrm{Kh}_{\mathrm{h}}$ in the proof of Theorem 1.5. He defined a strip $S(\alpha, \beta, \rho)$ for given $\alpha, \beta, \rho \in \mathrm{R}$ by

$$
S(\alpha, \beta, \rho)=\left\{x \in \mathrm{R}^{2} ; \alpha x_{1}+\beta-\rho(1+\alpha) \leq x_{2} \leq \alpha x_{1}+\beta+\rho(1+\alpha)\right\} .
$$

He called the number $\rho$ the diagonal half-width of the strip. The boundary of the strip consists of two components given by the lines $x_{2}=\alpha x_{1}+\beta \pm \rho(1+\alpha)$, i.e., the center line, $x_{2}=\alpha x_{1}+\beta$, translated by the vectors $(-\rho, \rho)$ and $(\rho,-\rho)$. As a consequence of the digitization of pure points, we can see easily that a set of pure points is a subset of a digital straight line segment if and only if they are contained in a strip with a diagonal half-width strictly less than $\frac{1}{2}$.

\section{Boomerangs and digital straight line segments}

In this paper we want to characterize the digital straight line segments, so we consider the collection of monotone functions on a bounded interval. We may restrict attention to monotone functions, because a function which is not monotone can never represent a straight line segment.

In the case of the Khalimsky topology, it is clear that the graph of a discontinuous function cannot have the chord property, so we do not need to consider such functions. We consider increasing functions; the case of decreasing functions is similar. For this case we have the chord property which we introduced in Definition 1.3.

If $P=\left(p^{i}\right)_{i=0}^{n}$ is a sequence of points which is the graph of a function $f$, thus with $p_{2}^{i}=f\left(p_{1}^{i}\right)$, we define its chain code $c=\left(c_{i}\right)_{i=1, \ldots, n}$ by $c_{i}=$ $f(i)-f(i-1), i=1, \ldots, n$. For the functions we work on, $c_{i}$ is equal to zero or one. (This definition agrees with the Freeman chain code in this case.)

The simplest straight line segments in the digital plane are the horizontal, diagonal and vertical ones. In the remaining cases the graph contains both horizontal and diagonal steps; we shall call them constant and increasing, respectively, so in this case we have at least one point preceded by a horizontal interval and followed by a diagonal interval, or conversely.

Definition 2.1. When a graph $P$ is given, we shall say that a digital curve consisting of $m+1$ points, $B=\left(b^{i}\right)_{i=0}^{m}, m \geq 2$, is a boomerang in $P$ if 
it consists of a horizontal segment $\left[b^{0}, b^{k}\right]$, where $0<k<m$, followed by a diagonal segment $\left[b^{k}, b^{m}\right]$, or conversely, and if $B$ is maximal with this property. We shall call the horizontal and diagonal segments, $\operatorname{Con}(B)$ and $\operatorname{lnc}(B)$, respectively.

We use $|\operatorname{Con}|=|\operatorname{Con}(B)|=k$ for the number of horizontal intervals in the segment $\left[b^{0}, b^{k}\right]$, and $|\operatorname{lnc}|=|\operatorname{Inc}(B)|=m-k$ for the number of diagonal intervals in the segment $\left[b^{k}, b^{m}\right]$, or conversely if the horizontal segment comes last. They are equal to the number of zeros and ones in the related chain code, respectively. We introduce $|B|=k+(m-k)=m$ as the sum of $|\operatorname{Con}(B)|$ and $|\operatorname{lnc}(B)|$. We remark that the boomerangs need not be disjoint and that the last segment of a boomerang may be a starting segment of the next boomerang, so the number of boomerangs is equal to the number of vertices.

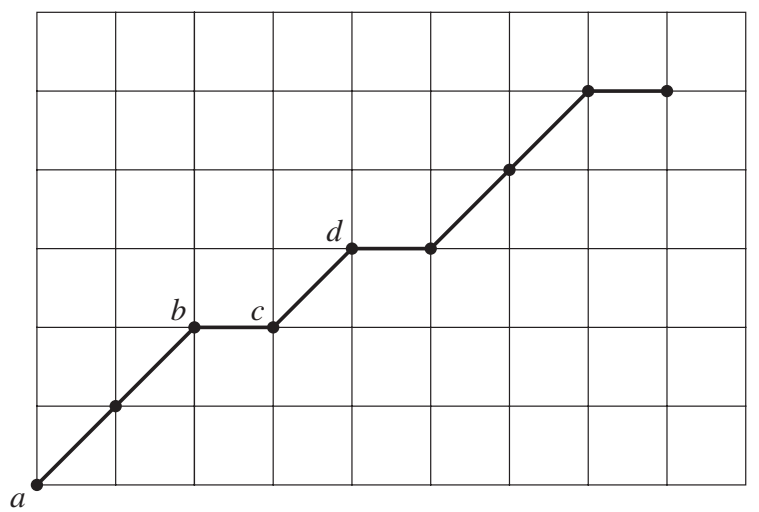

FIGURE 1.

A digital segment shown in figure 1 contains 5 boomerangs. The segments $a b$ and $b c$ is a concave boomerang with increasing part $a b$ and constant part $b c$. The vertex of this boomerang is the point $b$. The segment $b c$ together with the segment $c d$ show a convex boomerang.

We thus divide the collection of graphs of monotone functions on bounded intervals into two cases:

(I) Horizontal or diagonal;

(II) All others.

The case (I) is straightforward. We shall now discuss the second type of digital curves.

Definition 2.2. Given any subset $P$ of $\mathrm{R}^{2}$ we define its $\operatorname{chord}$ set $\operatorname{chord}(P)$ 
as the union of all chords, i.e., all segments with endpoints in $P$, as

$$
\operatorname{chord}(P)=\bigcup_{x, y \in P}[x, y] \subseteq \mathrm{R}^{2} .
$$

We also need the broken line defined for a finite sequence $P=\left(p^{i}\right)_{i=0}^{n}$,

$$
\mathrm{BL}(P)=\bigcup_{i=0}^{n-1}\left[p^{i}, p^{i+1}\right] \subseteq \mathrm{R}^{2} .
$$

Similarly for an infinite sequence $\left(p^{i}\right)_{i \in \mathrm{N}}$ or $\left(p^{i}\right)_{i \in \mathrm{Z}}$.

Lemma 2.3. For an 8 -connected sequence $P=\left(p^{i}\right)_{i=0}^{n}$ we have

$$
\mathrm{BL}(P)+B_{<}^{1}(0,1) \subseteq \bigcup_{i=0}^{n}\left(\left\{p^{i}\right\}+B_{<}^{\infty}(0,1)\right),
$$

where $\mathrm{BL}(P)+B_{<}^{1}(0,1)$ and $\left\{p^{i}\right\}+B_{<}^{\infty}(0,1)$ are the dilations of $\mathrm{BL}(P)$ and $\left\{p^{i}\right\}$ by the open unit ball for the $l^{1}$ and $l^{\infty}$ metric, respectively.

PRoOF. We can see easily that

$$
\begin{aligned}
\mathrm{BL}(P) & +B_{<}^{1}(0,1) \\
& =\bigcup_{i=1}^{n-1}\left[p^{i}, p^{i+1}\right]+B_{<}^{1}(0,1) \\
& \subseteq\left(\left\{p^{0}, p^{n}\right\}+B_{<}^{1}(0,1)\right) \cup\left(\bigcup_{i=0}^{n-1}\left[p^{i}, p^{i+1}\right]+\{0\} \times[-1,1]\right)
\end{aligned}
$$

We have

$$
\left\{p^{0}, p^{n}\right\}+B_{<}^{1}(0,1) \subseteq\left\{p^{0}, p^{n}\right\}+B_{<}^{\infty}(0,1)
$$

and

$$
\bigcup_{i=0}^{n-1}\left[p^{i}, p^{i+1}\right]+\{0\} \times[-1,1] \subseteq \bigcup_{i=0}^{n}\left\{p^{i}\right\}+B_{<}^{\infty}(0,1) .
$$

Then (3) and (4) give the result.

Remark 2.4. In the equation (2), if we consider an infinite sequence $P=$ $\left(p^{i}\right)_{i \in \mathrm{Z}}$, we have

$$
\bigcup_{i}\left[p^{i}, p^{i+1}\right]+B_{<}^{1}(0,1) \subseteq \bigcup_{i}\left(\left[p^{i}, p^{i+1}\right]+\{0\} \times[-1,1]\right) .
$$




\section{Boomerangs and vertical distance}

After introducing the notion of boomerang in Section 2, we shall study the relation of it with DSLS, $\mathrm{DSLS}_{\mathrm{Kh}}$ and vertical distances at some special points.

Suppose that $P=\left(p^{i}\right)_{i=0, \ldots, n}$ is a sequence of points which has $b$ boomerangs. Let $V=\left(v^{i}\right)_{i=1}^{b}$ be the sequence of all vertices of the boomerangs of $P$. We define the vertical distance $d_{\mathrm{v}}$ as $d_{\mathrm{v}}(x, y)=\left|x_{2}-y_{2}\right|$ when $x_{1}=y_{1}$. We shall show a relation between vertical distances and DSLS ${ }_{8}$ and $D S L S_{K h}$.

TheOREM 3.1. Let $P=\left(p^{i}\right)_{i=0}^{n}$ be an 8-connected sequence of points which is the graph of a function and has $b$ boomerangs. Let $V=\left(v^{i}\right)_{i=1, \ldots, b}$ be the sequence of all vertices of its boomerangs. Then $P \in \mathrm{DSLS}_{8}$ if and only if for all $i=1, \ldots, b$ and all real points $a \in \operatorname{chord}(P)$ such that $a_{1}=v_{1}^{i}$ we have $d_{\mathrm{v}}\left(v^{i}, a\right)<1$.

Proof. Suppose that there is a vertex $v=p^{j}$ for some $0<j<n$ and a point $a \in \operatorname{chord}(P)$ with $a_{1}=v_{1}$ such that $d_{\mathrm{v}}(v, a) \geq 1$. We shall show that $P \notin \mathrm{DSLS}_{8}$. Since we have $d_{\mathrm{v}}(v, a) \geq 1$,

$$
a \notin\{v\}+B_{<}^{\infty}(0,1) .
$$

Also

$$
\left|a_{1}-p_{1}^{i}\right| \geq 1 \quad \text { for } \quad i \neq j .
$$

Therefore, by (5), (6), we see that

$$
a \notin\left\{p^{i}\right\}_{i=0}^{n}+B_{<}^{\infty}(0,1),
$$

and so $P \notin \mathrm{DSLS}$.

Conversely, suppose that $P \notin \mathrm{DSLS}$, so there is a point $c$ and two indices $k, l$ such that $0 \leq k<l \leq n$ and $c \in\left[p^{k}, p^{l}\right]$ but $c \notin P+B_{<}^{\infty}(0,1)$. By Lemma 2.3,

$$
c \notin \mathrm{BL}(P)+B_{<}^{1}(0,1) .
$$

Define $Q_{k, l}=\mathrm{BL}\left(\left(p^{i}\right)_{i=k, \ldots, l}\right)$. Consider the function $F_{k, l}:\left[p_{k}, p_{l}\right] \rightarrow \mathrm{R}$ defined by

$$
F_{k, l}(x)=d_{\mathrm{v}}(x, y) \quad \text { for } \quad y \in Q_{k, l} \text { with } y_{1}=x_{1} .
$$

Consider the point $x \in Q_{k, l}$ with $x_{1}=c_{1}$. By (7),

$$
d_{\mathrm{v}}(c, x) \geq 1 \text {. }
$$


Therefore $F_{k, l}(c) \geq 1$. The function $F_{k, l}$ attains its maximum at a point that lies on a vertical line passing through a vertex, so there is a vertex $v$ of the boomerang $B$ such that the function $F_{k, l}$ attains its maximum at the point $a \in\left[p^{k}, p^{l}\right]$ with $a_{1}=v_{1}$, thus

$$
1 \leq F_{k, l}(c) \leq F_{k, l}(a)=d_{\mathrm{v}}(v, a) .
$$

This shows that, for the vertex $v$ and a point $a \in \operatorname{chord}(P)$ with same first coordinate as $v$, we have $d_{\mathrm{v}}(a, v) \geq 1$. We are done.

We shall now study the same result for Khalimsky-connectedness. We consider mixed points $m=\left(m_{1}, m_{2}\right)$ which lie on $P$ and such that for some vertex $v=\left(v_{1}, v_{2}\right)$, we have $m_{1}=v_{1} \pm 1$. In the next theorem we shall show that we have straightness if and only if the vertical distance is less than one at these mixed points.

Theorem 3.2. Suppose that $P=\left(p^{i}\right)_{i=0}^{n}$ is a Khalimsky-connected sequence with pure endpoints and let $b$ be the number of its boomerangs. Let $M$ be the set of all mixed points in $P$. Then $P \in \mathrm{DSLS}_{\mathrm{Kh}}$ if and only if for all $m \in M$ and all $a \in \operatorname{chord}(P)$ with $a_{1}=m_{1}$ we have $d_{\mathrm{v}}(m, a)<1$.

Proof. Suppose that there exist a mixed point $m=p^{j}$ for some $0<j<n$ and a point $a \in \operatorname{chord}(P)$ with $a_{1}=m_{1}$ such that $d_{\mathrm{v}}(m, a) \geq 1$, so that

$$
a \notin\{m\}+B_{<}^{\delta^{\infty}}(0,1),
$$

where $B_{<}^{\delta^{\infty}}(0,1)$ is the open unit ball for the metric $\delta^{\infty}$. We shall obtain a contradiction.

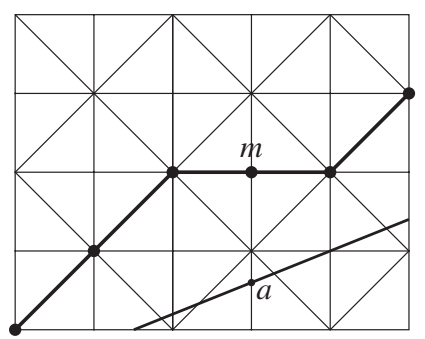

FIGURE 2. The mixed point $m=p^{j}$ and point $a \in \operatorname{chord}(P)$ with $a_{1}=m_{1}$ and $d_{\mathrm{v}}(m, a) \geq 1$, leading to a contradiction in the proof.

It is clear that

$$
\left|a_{1}-p_{1}^{j-2}\right|=2 \quad \text { and } \quad\left|a_{1}-p_{1}^{j+2}\right|=2 ;
$$




$$
\left|a_{1}-p_{1}^{k}\right| \geq 2 \quad \text { for } \quad k \geq j+2 \text { and } k \leq j-2 .
$$

We can see easily also that

$$
\left|a_{2}-p_{2}^{j-1}\right|=\left|a_{2}-p_{2}^{j+1}\right| \geq 1 .
$$

Therefore, by (8), (9) and (10)

$$
a \notin P+B_{<}^{\delta^{\infty}}(0,1) .
$$

Thus $P \notin \mathrm{DSLS}_{\mathrm{Kh}}$.

Conversely, suppose that $P \notin \mathrm{DSLS}$ Kh, so that we can find a point $c$ and two indices $k, l$ such that $0 \leq k<l \leq n$ and $c \in\left[p^{k}, p^{l}\right]$ but $c \notin P+B_{<}^{\delta^{\infty}}(0,1)$. Let us assume that $c$ is below the graph of $P$. The boundary of $P+B_{<}^{\delta^{\infty}}(0,1)$ consists of horizontal and vertical segments. We can choose a point $b$ on the line segment $\left[p^{k}, p^{l}\right]$ such that $b \notin P+B_{<}^{\delta^{\infty}}(0,1)$ and $b$ has an integer first coordinate.

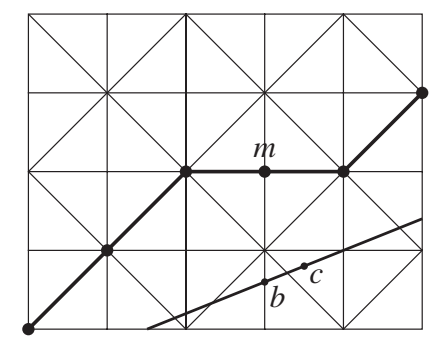

Figure 3. Two points $b, c \in \operatorname{chord}(P)$ which do not belong to $P+B_{<}^{\delta^{\infty}}(0,1)$ and where the vertical distance at the mixed point $m$ is greater than or equal to one. This leads to a contradiction in the proof.

As we can see in Figure 3, this can achieved by taking a point on the segment not belonging to $P+B_{<}^{\delta^{\infty}}(0,1)$ and then going to the left as far as possible to the intersection of the line segment $\left[p^{k}, p^{l}\right]$ and a vertical segment in the boundary of $P+B_{<}^{\delta^{\infty}}(0,1)$. The straight line defined by this vertical segment contains a point $m=\left(m_{1}, m_{2}\right) \in P$; thus $m_{1}=b_{1}$. Now $m$ must be a mixed point in $M$, because the neighboring point $\left(m_{1}-1, m_{2}\right)$ is the vertex of a boomerang and hence must be pure. Since $b$ lies on the boundary of the open set $P+B_{<}^{\delta^{\infty}}(0,1)$, we can conclude that $d_{\mathrm{v}}(m, b) \geq 1$.

According to the result of this section, using vertical distances for some points, makes straightness easier to check for both 8-connectedness and the Khalimsky plane. 


\section{Boomerangs and straightness}

We shall now discuss straightness by considering boomerangs and using the conditions on vertical distances in Theorems 3.1 and 3.2. First we just consider one boomerang. In two lemmas we shall find conditions for straightness in the 8-connected case and the Khalimsky case, and then we shall do the same when we have more than one boomerang.

Lemma 4.1. Let $B=\left(b^{i}\right)_{i=0}^{n}$ be an 8-connected boomerang. Then the following two properties are equivalent.

(i) $B \in \mathrm{DSLS}_{8}$;

(ii) If $|\operatorname{Con}(B)| \geq 2$, then $|\operatorname{Inc}(B)|=1$.

Proof. (i) $\Rightarrow$ (ii). Suppose that a boomerang $B \in \mathrm{DSLS}_{8}$ and $\mid$ Con $\mid \geq 2$ and $|\operatorname{lnc}| \geq 2$. Therefore the vertical distance between the vertex of $B$ and $\operatorname{chord}(B)$ is at least one. Theorem 3.1 now gives a contradiction.

(ii) $\Rightarrow$ (i). Suppose that $|\operatorname{lnc}|=1$, and that $\mid$ Con $\mid=m \geq 2$. We can check easily the condition Theorem 3.1 and see that $B \in \mathrm{DSLS}_{8}$.

Lemma 4.2. Let a boomerang $B=\left(b^{i}\right)_{i=0}^{n}$ be a Khalimsky-connected set with pure end points. Then the following two properties are equivalent.

(i) $B \in \mathrm{DSLS}_{\mathrm{Kh}}$;

(ii) If $|\operatorname{Con}(B)| \geq 4$, then $|\operatorname{lnc}(B)|=1$.

Proof. (i) $\Rightarrow$ (ii). Suppose that $B \in \mathrm{DSLS}_{\mathrm{Kh}}$ and $\mid$ Con $\mid \geq 4$ and $|\operatorname{Inc}| \geq 2$. By Theorem 3.2, we have contradiction.

(ii) $\Rightarrow$ (i). Suppose that $|\operatorname{Inc}|=1$, and $\mid$ Con $\mid \geq 4$. We can see easily that the condition in Theorem 3.2 is satisfied, and we are done.

The two previous Lemmas 4.1 and 4.2 show the relation between the class DSLS and an arbitrary boomerang, but of course there are digital curves such that all its constituent boomerangs satisfy the condition of these lemmas but the curve itself is not in DSLS. In order to avoid complicated proofs in Propositions 4.3 and 4.4 and Lemmas 4.6 and 4.7, or a complicated statement in Theorem 4.8 , we will consider only concave boomerangs.

Proposition 4.3. Suppose that $P=\left(p^{i}\right)_{i=0}^{n}$ is a set of points such that $P \in \mathrm{DSLS}_{8}$ and denote by $b$ the number of concave boomerangs in $P$. If $\left|\operatorname{Con}\left(B_{j}\right)\right| \geq 2$ for some $j$ with $1 \leq j \leq b$, then $\left|\operatorname{Inc}\left(B_{i}\right)\right|=1$ for all $i$ with $1 \leq i \leq b$.

Proof. Let $P \in \mathrm{DSLS}_{8}$. Suppose that there exist $1 \leq i, j \leq b$ such that $\left|\operatorname{lnc}\left(B_{i}\right)\right| \geq 2$ and $\left|\operatorname{Con}\left(B_{j}\right)\right| \geq 2$. It suffices to study the case $i \leq j$. We may assume that $\left|\operatorname{lnc}\left(B_{i}\right)\right|=2,\left|\operatorname{Con}\left(B_{j}\right)\right|=2$ by passing to subsets and $B_{i}$ is the 
closest boomerang to $B_{j}$ with cardinality of the increasing part not equal to 1 . If $i=j$, the result is obvious by Lemma 4.1. For $j-i=1$, by Lemma 4.1 we must have $\left|\operatorname{Con}\left(B_{i}\right)\right|=\left|\operatorname{Inc}\left(B_{j}\right)\right|=1$. By Theorem 3.1 we do not have straightness in this case.

Suppose now that $j-i>1$. In this case the chain code for $P$ is

$$
\left(1,1,0,(1,0)^{t}, 1,0,0\right)
$$

where $(1,0)^{t}$ means that we have $t$ times the subsequence $(1,0)$. Let $\left(p^{i}\right)_{i=l}^{l+2 t+6}$ be the points related to this chain code. The slope of the line segment $\left[p^{l}\right.$, $\left.p^{l+2 t+6}\right]$ is equal to $\frac{3+t}{6+2 t}=\frac{1}{2}$. We can check easily that the vertical distance between the vertex $p^{l+2}$ and the line segment $\left[p^{l}, p^{l+2 t+6}\right]$ is 1 . Thus we are done just by considering Theorem 3.1.

Proposition 4.4. Suppose that $P=\left(p^{i}\right)_{i=0}^{n}$ is a Khalimsky-connected sequence with pure endpoints such that $P \in \mathrm{DSLS}_{\mathrm{Kh}}$ and denote by $b$ the number of concave boomerangs in $P$. If $\left|\operatorname{Con}\left(B_{j}\right)\right| \geq 4$ for some $1 \leq j \leq b$, then $\left|\operatorname{Inc}\left(B_{i}\right)\right|=1$ for all $1 \leq i \leq b$.

Proof. We do as in the proof of Proposition 4.3. Suppose that there exist $1 \leq i \leq j \leq b$ such that $\left|\operatorname{lnc}\left(B_{i}\right)\right| \geq 2$ and $\left|\operatorname{Con}\left(B_{j}\right)\right| \geq 4$. We may assume that $\left|\operatorname{Inc}\left(B_{i}\right)\right|=2,\left|\operatorname{Con}\left(B_{j}\right)\right|=4$ by passing to subsets. We can assume that $B_{i}$ is the closest boomerang to $B_{j}$ with cardinality of the increasing part not equal to 1. For $j-i=1$, we can find a contradiction as in Proposition 4.3. Finally, we shall show that we do not have straightness when $j-i>1$. Let $\left(1,1,0,0,(1,0,0)^{t}, 1,0,0,0,0\right)$ be the related chain code for the set of boomerangs $B_{i}, \ldots, B_{j}$ and $\left(p^{i}\right)_{i=l}^{l+3 t+9}$ be the points related to this chain code. The slope of the line segment $\left[p^{l}, p^{l+3 t+9}\right]$ is equal to $\frac{3+t}{9+3 t}=\frac{1}{3}$. Thus, we can see that the vertical distance between the mixed point $p^{l+3}$ and the line segment $\left[p^{l}, p^{l+3 t+9}\right]$ is equal to 1 . Therefore, we do not have straightness by Theorem 3.2.

By Propositions 4.3 and 4.4, there are just two cases when we study straightness. We write them in the following definition.

Definition 4.5. Let $I_{i}=\left|\operatorname{Inc}\left(B_{i}\right)\right|$ and $C_{i}=\left|\operatorname{Con}\left(B_{i}\right)\right|$, where $1 \leq i \leq b$ and $b$ is the number of boomerangs in $P$. We shall consider four cases:

(8-a) $I_{i}=1$ for all $1 \leq i \leq b$;

(8-b) $C_{i}=1$ for all $1 \leq i \leq b$;

(Kh-a) $I_{i}=1$ for all $1 \leq i \leq b$;

(Kh-b) $C_{i}=2$ for all $1 \leq i \leq b$. 
We shall call $P$ dominant constant if it satisfies condition (8-a) in the case of 8-connectedness, and condition (Kh-a) in the case of Khalimsky connectedness, and dominant increasing if it satisfies condition (8-b) in the case of 8-connectedness and condition (Kh-b) in the case of Khalimsky connectedness.

If the discrete straight line has slope between 0 and $\frac{1}{2}$, we have dominant constant and for the slope of the line between $\frac{1}{2}$ and 1 , we have dominant increasing.

There are some results on the runs of 8-connected digital straight lines that are related to our work. We give a summary of them. Freeman [3] has observed that (except possibly at the beginning and end of the segment) the "successive occurrencies of the element occurring singly are as uniformly spaced as possible."

Rosenfeld [16] provided a formal proof of these facts for the 8-connected case. We present two propositions, in the 8-connected case and the Khalimskyconnected case with this conclusion. We shall show that we have two possibilities for the number of boomerangs in both cases. This result is similar to Rosenfeld's conclusion in the 8-connected case for runs. We shall use the results of these lemmas in Theorem 4.8, so we write the statements of the two lemmas using boomerangs. To prove these lemmas we shall use Theorems 3.1 and 3.2.

Lemma 4.6. If $P \in \mathrm{DS} \mathrm{LS}_{8}$, then we have at most two possible values for the cardinality of the boomerangs in $P$, that is, ||$B_{i+k}|-| B_{i}|| \leq 1$ for all $i, k \in \mathrm{N}$.

Proof. Let $P$ be dominant increasing. To avoid complicated indices and to simplify the construction of the proof, we consider concave boomerangs only. We choose $k$ minimal such that

$$
|| B_{i+j}|-| B_{i}||=1 \quad \text { for } \quad 1 \leq j<k,
$$

and

$$
|| B_{i+k}|-| B_{i}|| \geq 2 \text {. }
$$

Without loss of generality, we may assume that $\left|B_{i+j}\right| \geq\left|B_{i}\right|$ for $1 \leq j \leq k$. Thus

$$
\left|B_{i+j}\right|-\left|B_{i}\right|=1 \quad \text { for } \quad 1 \leq j<k,
$$

and

$$
\left|B_{i+k}\right|-\left|B_{i}\right| \geq 2 .
$$

Consider now the line segment $[p, q]$ such that $p$ is the starting point of $\operatorname{Con}\left(B_{i-1}\right)$ and $q$ is the endpoint of $\operatorname{Inc}\left(B_{i+k}\right)$. This line segment has slope

$$
\frac{(k+1) I_{i}+k-1+t}{(k+1) I_{i}+2 k+t},
$$


where

$$
t=\left|B_{i+k}\right|-\left|B_{i}\right| \geq 2 \quad \text { and } \quad I_{i}=\left|\operatorname{lnc}\left(B_{i}\right)\right| .
$$

We can see easily that the vertical distance is at least one at the point $\left(I_{i}+2, I_{i}\right)$ (which is the vertex of a convex boomerang). Therefore, we get a contradiction by Theorem 3.1. The proof for dominant constant can be obtained in the same way.

LEMMA 4.7. If $P \in \mathrm{DSLS}_{\mathrm{Kh}}$, then we have two possible values for the cardinality of boomerangs in $P$, that is, in the dominant increasing case,

$$
|| B_{i+k}|-| B_{i}|| \leq 1 \quad \text { for all } k \in \mathrm{N}
$$

and in the dominant constant case,

$$
|| B_{i+k}|-| B_{i}|| \leq 2 \quad \text { for all } \quad k \in \mathrm{N}
$$

Proof. For the dominant increasing, we do as in Lemma 4.6. Here we consider, as in Lemma 4.6, concave boomerangs. We choose $k$ minimal such that

$$
\left|B_{i+j}\right|-\left|B_{i}\right|=1 \quad \text { for } \quad 1 \leq j<k,
$$

and

$$
\left|B_{i+k}\right|-\left|B_{i}\right| \geq 2
$$

Consider the line segment $[p, q]$ such that $p$ is the starting point of $\operatorname{Con}\left(B_{i-1}\right)$ and $q$ is the endpoint of $\operatorname{Inc}\left(B_{i+k}\right)$. This line segment has slope

$$
\frac{(k+1) I_{i}+k-1+t}{(k+1) I_{i}+3 k+t+1},
$$

where

$$
t=\left|B_{i+k}\right|-\left|B_{i}\right| \geq 2 \quad \text { and } \quad I_{i}=\left|\operatorname{lnc}\left(B_{i}\right)\right| .
$$

We can see easily that the vertical distance is at least one at the mixed point $\left(I_{i}+3, I_{i}\right)$. Thus, we are done for the dominant increasing case by getting a contradiction with Theorem 3.2.

Suppose now that $P$ is dominant constant. We may choose $k$ minimal such that

$$
\left|B_{i+j}\right|-\left|B_{i}\right|=2 \quad \text { for } \quad 1 \leq j<k,
$$

and

$$
\left|B_{i+k}\right|-\left|B_{i}\right| \geq 4
$$

Consider the line segment $[p, q]$ where $p$ and $q$ are the start point of $\operatorname{Inc}\left(B_{i}\right)$ and the endpoint of Con $\left(B_{i+k}\right)$, respectively. We can easily check that the vertical 
distance is at least one at the mixed point $\left(C_{i}+3,2\right)$, where $C_{i}=\left|\operatorname{Con}\left(B_{i}\right)\right|$. Thus, considering Theorem 3.2, we get a contradiction.

The conditions in Lemmas 4.6 and 4.7 are necessary but not sufficient for straightness. An example for this claim is the set of 8-connected points with Freeman chain code 11010110101010 . These points satisfy the conclusion of Lemma 4.6 but do not have the chord property. In the Khalimsky plane we can see these results in the set of points with Freeman chain code 11001001100100100100.

Hung and Kasvand [4] introduced a way to find the sufficient condition for straightness in the 8-connected plane. They considered a digital arc as a sequence of two symbols. Then they noted that a segment in a sequence of symbols is a continuous block of symbols of this sequence; the number of symbols in a segment is the length of this segment. All segments having the same length in a sequence were called equal segments. Two equal segments they called uneven if their sums differ by more than 1 . They called any two uneven segments an uneven pair. Then they went on to prove that a digital arc has the chord property if and only if there are no uneven segments in its chain code. They named a digital arc straight if and only if for equal segments in this arc, their sums cannot differ by more than 1 . Therefore, like the chord property, the absence of uneven segments is one of the most fundamental properties in the structure of a digital straight line.

Bruckstein [2] presented several interesting self-similarity properties of chain codes of digital straight line. He introduced some transformations given by matrices of determinant \pm 1 . These matrices belong to the well-known group $\mathrm{GL}(2, \mathrm{Z})$. As a result of these transformations, he showed that the new sequence produced by applying these transformation to a sequence of 0 and 1 is the chain code of digital straight line segment if and only if the original sequence is the chain code of a digital straight line segment.

To find a sufficient condition for straightness, we shall define a mapping which transforms certain codes to the set $\{0,1\}$. Let $\mathrm{B}(P)$ be the collection of all boomerangs in $P$. By Lemmas 4.6 and 4.7, we have just two possibilities for the values of $\left|B_{i}\right|$. Thus we can define a mapping from the set of Freeman chain code of $P$ to $\{0,1\}$ which maps the boomerangs with greater cardinality to 1 and the other boomerangs to 0 . The graph of $f$ is an 8-connected set and by this fact we can see easily that if $P$ is a Khalimsky-connected set, then $f(P)$ will be an 8-connected set and so for investigating the straightness in Khalimsky plane we can go to the 8-connected case. In the following theorem we shall show that $f(P)$ and so the composition of $f$ with itself can give a necessary and sufficient condition for straightness in the 8-connected case and therefore also in the Khalimsky-connected plane. 
THEOREM 4.8. We define a function $f$ on a subset of the set $\{0,1\}^{\mathrm{N}}$ of sequences of zeros and ones and with values in the same set: $f(C)$ is defined for those chain codes that represent dominant increasing or dominant constant sequences which arise from sets of boomerangs of at most two different lengths. We define $f(C)$ as the sequence obtained by replacing the chain code of a long concave boomerang by 1 and that of a short concave boomerang by 0 . Then

(I) $C$ is the chain code of an element of $\mathrm{DSLS}_{8}$ if and only if $f(C) \in \mathrm{DSLS}_{8}$, and

(II) $C$ is the chain code of an element of $\mathrm{DSLS} \mathrm{Kh}_{\mathrm{Kh}}$ if and only if $C$ is the chain code of a Khalimsky-connected set and $f(C) \in \mathrm{DSLS}_{8}$.

Remark 4.9. If we compose $f$ with itself and define $f^{0}(C)=C$, $f^{n+1}(C)=f\left(f^{n}(C)\right)$ for $n \in \mathrm{N}$, then $f^{n}(C)$ belongs to $\mathrm{DSLS}_{8}$ for all $n \in \mathrm{N}$ and all $C \in \mathrm{DSLS}_{8}$, and $f^{n}(C)$ belongs to DSLS for all $n \in \mathrm{N}^{*}=\mathrm{N} \backslash\{0\}$ for all $C \in \mathrm{DSLS}_{\mathrm{Kh}}$.

Proof. We define a transformation which gives us the chain code of $f(C)$. We want to transform a short boomerang to a vector $V_{1}$ which comes from the line segment between the starting point and the endpoint of this boomerang. Then, in analogy with short boomerangs we can do the same for a long boomerang and transform it to a vector $V_{3}$. We define a grid $T$ which is contained in $\mathrm{R}^{2}$ and has two linearly independent basis vectors $V_{1}$ and $V_{2}$, where $V_{2}$ is the sum of $V_{1}$ and $V_{3}$. Therefore

$$
T=\left\{a+x_{1} V_{1}+x_{2} V_{2} ; x=\left(x_{1}, x_{2}\right) \in Z^{2}\right\} \quad \text { with } a=\left(a_{1}, a_{2}\right) \text { as origin. }
$$

With this transformation, we can map the set chord $(C)$ into $\mathrm{R}^{2}$. The image of $x=\left(x_{1}, x_{2}\right)^{\mathrm{T}}=\left(\begin{array}{c}x_{1} \\ x_{2}\end{array}\right) \in \mathrm{Z}^{2}$ in $T$ is

$$
\left(\begin{array}{l}
t_{1} \\
t_{2}
\end{array}\right)=\left(\begin{array}{l}
a_{1} \\
a_{2}
\end{array}\right)+A\left(\begin{array}{l}
x_{1} \\
x_{2}
\end{array}\right)
$$

where

$$
A=\left(\begin{array}{cc}
1 & -1 \\
1-p & p
\end{array}\right) \quad \text { or } \quad A=\left(\begin{array}{cc}
0 & 1 \\
1 & -p
\end{array}\right)
$$

for the set of 8-connected points which is dominant increasing or dominant constant, respectively, and $p$ denotes the cardinality of a short boomerang.

In the same way we define a transformation which gives us the chain code of $f(C)$ in the Khalimsky case. We notice that in the dominant increasing case, the constant part is always 2 and in the dominant constant case, the constant part 
must be an even number. We can write this transformation in the Khalimsky case using a matrix $A$ defined as follows:

$$
A=\frac{1}{2}\left(\begin{array}{cc}
1 & -1 \\
2-p & p
\end{array}\right) \quad \text { or } \quad A=\left(\begin{array}{cc}
0 & 1 \\
\frac{1}{2} & -\frac{1}{2} p
\end{array}\right)
$$

for the set of points which is dominant increasing or dominant constant, respectively. The number $p$ is the cardinality of the short boomerangs, which is an odd number for the dominant constant case. In both cases we can come back from $T$ to $Z^{2}$ as follows:

$$
\left(\begin{array}{l}
x_{1} \\
x_{2}
\end{array}\right)=A^{-1}\left[\left(\begin{array}{l}
t_{1} \\
t_{2}
\end{array}\right)-\left(\begin{array}{l}
a_{1} \\
a_{2}
\end{array}\right)\right] .
$$

By the statement of Theorem 4.8, $C$ is dominant increasing or dominant constant. If $f(C)$ is a digital straight line segment, then we have four possibilities in each of the two cases, the 8-connected case and the Khalimsky case. We present them in the following list.

1. $C$ is dominant increasing and $f(C)$ is dominant increasing, so $C$ has dominant long boomerangs;

2. $C$ is dominant constant and $f(C)$ is dominant increasing, so $C$ has dominant long boomerangs;

3. $C$ is dominant increasing and $f(C)$ is dominant constant, so $C$ has dominant short boomerangs;

4. $C$ is dominant constant and $f(C)$ is dominant constant, so $C$ has dominant short boomerangs.

In the case of 8-connectedness, there are no special differences in the proof of the four cases in (14), but in the Khalimsky case, we must be careful which possibility we choose to work on, and how we can transform a mixed point to a vertex and vice versa.

Case (I), $\Rightarrow$. Now we shall prove the implication $\Rightarrow$ in case (I). Let $C \in$ $\mathrm{DSLS}_{8}$. If $f(C) \notin \mathrm{DSLS} S_{8}$ then we can find a vertex $v=\left(v_{1}, v_{2}\right)^{\mathrm{T}}$ of a boomerang $B$ such that we have vertical distance at least one at this point. Suppose that this vertical distance is attained between $v$ and the line segment with equation $Y=M X+N$ in $T$. Thus

$$
d_{\mathrm{v}}(v, a)=v_{2}-M v_{1}-N \geq 1 .
$$

Since we exclude convex boomerangs in this section, we can find easily the vertical distances without considering the absolute value. We may assume that 
$C$ is dominant increasing. The transformation of the vertex $v$ into $Z^{2}$ is an endpoint of a boomerang in $C$. Let $v^{\prime}$ be this image. Thus

$$
v^{\prime}=\left(\begin{array}{cc}
p & 1 \\
p-1 & 1
\end{array}\right)\left(\begin{array}{l}
v_{1} \\
v_{2}
\end{array}\right)=\left(\begin{array}{c}
p v_{1}+v_{2} \\
(p-1) v_{1}+v_{2}
\end{array}\right) .
$$

To find the image of the straight line $Y=M X+N$ in $\mathrm{Z}^{2}$, we do as follows:

$$
\left(\begin{array}{l}
x \\
y
\end{array}\right)=\left(\begin{array}{cc}
p & 1 \\
p-1 & 1
\end{array}\right)\left(\begin{array}{l}
X \\
Y
\end{array}\right) .
$$

By (15) and a simple calculation,

$$
\begin{aligned}
& x=(p+M) X+N, \\
& y=(p-1+M) X+N .
\end{aligned}
$$

This implies

$$
y=\frac{M+p-1}{M+p} x+\frac{N}{M+p} .
$$

Thus the vertical distance between the line segment with equation (16) and the vertex $\left(p v_{1}+v_{2}-1,(p-1) v_{1}+v_{2}\right)^{\mathrm{T}}$ is

$$
\begin{aligned}
(p-1) v_{1}+v_{2}-\frac{M+p-1}{M+p} & \left(p v_{1}+v_{2}-1\right)-\frac{N}{M+p} \\
= & \frac{\left(v_{2}-M v_{1}-N\right)+(M+p-1)}{M+p} \geq 1 .
\end{aligned}
$$

By Theorem 3.1, we can conclude that $C \notin \mathrm{DSLS}_{8}$. That is a contradiction. Therefore, the assertion is proved when $C$ is dominant increasing. The proof is similar for the dominant constant case.

Case $(\mathrm{I}), \Leftarrow$. Conversely, we shall now prove the implication $\Leftarrow$ in case (I). Let $f(C)$ be in $\mathrm{DSLS}_{8}$. By the statement of this Theorem, $C$ must be dominant increasing or dominant constant. We have two possibilities for the cardinalities of boomerangs. By (14) we have four possibilities and the proof for those we use the same construction. We must consider the matrix for the transformation with the construction of $C$ as dominant increasing or dominant constant. Assume now we are in case 1 in (14). Thus the sequences $C$ and $f(C)$ are dominant increasing and $C$ has dominant long boomerangs. Suppose that $C \notin \mathrm{DSLS}_{8}$. Then by Theorem 3.1 we can find a vertex $v$ of a boomerang $B$ such that the vertical distance between this vertex and $\operatorname{chord}(C)$ is at least one. First, we shall show that the maximal vertical distance in $C$ is attained 
at a vertex $v$ of a long boomerang, where the following boomerang is short. Let $\left(B_{l}, \ldots, B_{l+k}\right)$ be the set of all long boomerangs which lie between two short boomerangs and such that there is no short boomerang between them. Consider the line segment $[a, b]$ with equation $y=\alpha x+\beta$ in the $\operatorname{chord}(C)$ such that the maximal vertical distance is attained between this line segment and the vertex $v$. The point $a$ must be the starting point of a boomerang and $b$ the endpoint of another boomerang. Thus the slope of this line segment is

$$
\alpha=\frac{(r+s) p-s}{(r+s) p+r},
$$

where $r$ and $s$ are the number of long and short boomerangs, respectively. By a simple calculation, we can see that the condition for the maximal vertical distance to be attained at the vertex of $B_{l+k}$ is

$$
\frac{p-1}{p} \leq \alpha \leq \frac{p}{p+1}
$$

We can check that the inequality (18) is correct by using (17). By the previous discussion, the vertex $v$ must be the vertex of the last boomerang, i.e., $B_{l+k}$. Since $\left(B_{l}, \ldots, B_{l+k}\right)$ are long boomerangs and $B_{l+k+1}$ is a short boomerang, the image of $\left(B_{l}, \ldots, B_{l+k}, B_{k+l+1}\right)$ in $T$ is a boomerang with its vertex equal to the image of the endpoint of $B_{l+k}$ in $T$. By the previous discussion, the maximal vertical distance is attained at the vertex $v=\left(v_{1}, v_{2}\right)^{\mathrm{T}}$ of the boomerang $B_{l+k}$. So that the point $a$ with the same first coordinate as $v$ and which lies on the line segment $y=\alpha x+\beta$ satisfies

$$
d_{\mathrm{v}}(v, a)=v_{2}-\alpha v_{1}-\beta \geq 1 .
$$

Since $C$ is dominant increasing, the endpoint of $B_{l+k}$ is $q=\left(v_{1}+1, v_{2}\right)^{\mathrm{T}}$. The image of $q$ in $T$ is

$$
q^{\prime}=\left(\begin{array}{cc}
1 & -1 \\
1-p & p
\end{array}\right)\left(\begin{array}{c}
v_{1}+1 \\
v_{2}
\end{array}\right)=\left(\begin{array}{c}
v_{1}-v_{2}+1 \\
(1-p)\left(v_{1}+1\right)+p v_{2}
\end{array}\right)
$$

that is, the vertex of the boomerang $B$ in $T$. The image of a line segment with equation $y=\alpha x+\beta$ in $T$ is

$$
Y=\frac{\alpha p-p+1}{1-\alpha} X+\frac{\beta}{1-\alpha},
$$

so the vertical distance between this line segment and $q^{\prime}$ is

$$
(1-p)\left(v_{1}+1\right)+p v_{2}-\frac{\alpha p-p+1}{1-\alpha}\left(v_{1}-v_{2}+1\right)-\frac{\beta}{1-\alpha}
$$




$$
=\frac{v_{2}-\alpha v_{1}-\beta-\alpha}{1-\alpha} \geq \frac{1-\alpha}{1-\alpha}=1 .
$$

Finally, by considering Theorem 3.1, we get a contradiction. For case 3 in (14), the maximal vertical distance is attained at the vertex of a long boomerang where the following boomerang is short. In case 2 [4] we have maximal vertical distance at the vertex of a long [long] boomerang where the previous boomerang is short [short]. We can prove these facts in the same way as in case 1. The proofs for straightness in these cases are also similar to that of case 1 .

Case (II), $\Rightarrow$. We shall now prove the implication $\Rightarrow$ in case (II). Let $C \in \mathrm{DSLS}_{\mathrm{Kh}}$. If $f(C) \notin \mathrm{DSLS}_{8}$ then we can find a vertex $v=\left(v_{1}, v_{2}\right)^{\mathrm{T}}$ of a boomerang $B$ and the line segment with equation $Y=M X+N$ in $T$ such that for the point $a$ which lies on this line segment and has the same first coordinate as $v$, the vertical distance is at least one. Thus

$$
d_{\mathrm{v}}(v, a)=v_{2}-M v_{1}-N \geq 1 .
$$

Suppose that $C$ is dominant increasing. The transformation of the vertex $v$ into $\mathrm{Z}^{2}$ is an endpoint of a boomerang in $C$. Let $v^{\prime}$ be this image. Thus

$$
v^{\prime}=\left(\begin{array}{cc}
p & 1 \\
p-2 & 1
\end{array}\right)\left(\begin{array}{l}
v_{1} \\
v_{2}
\end{array}\right)=\left(\begin{array}{c}
p v_{1}+v_{2} \\
(p-2) v_{1}+v_{2}
\end{array}\right) \text {. }
$$

The image of the straight line $Y=M X+N$ in $\mathrm{Z}^{2}$ is

$$
y=\frac{M+p-2}{M+p} x+\frac{2 N}{M+p} .
$$

The point $m=\left(p v_{1}+v_{2}-1,(p-2) v_{1}+v_{2}\right)^{\mathrm{T}}$ is a mixed point in a boomerang in $C$. Thus the vertical distance between the line segment with equation (19) and the mixed point $m$ is

$$
\begin{aligned}
(p-2) & v_{1}+v_{2}-\frac{M+p-2}{M+p}\left(p v_{1}+v_{2}-1\right)-\frac{2 N}{M+p} \\
= & \frac{\left(2 v_{2}-2 M v_{1}-2 N\right)+(M+p-2)}{M+p} \geq \frac{2+M+p-2}{M+p}=1 .
\end{aligned}
$$

By Theorem 3.2; $C \notin \mathrm{DSLS}_{\mathrm{Kh}}$. That is a contradiction.

Suppose now $C$ is dominant constant. The image of the vertex $v$ in $Z^{2}$ is:

$$
v^{\prime}=\left(\begin{array}{ll}
p & 2 \\
1 & 0
\end{array}\right)\left(\begin{array}{l}
v_{1} \\
v_{2}
\end{array}\right)=\left(\begin{array}{c}
p v_{1}+2 v_{2} \\
v_{1}
\end{array}\right)
$$


Without loss of generality, we can assume that the vertex $v$ is the vertex of a convex boomerang. Thus, the vertical distance between this point and the line segment with equation $Y=M X+N$ is $M v_{1}+N-v_{2}$, which is at least one. Same as previous discussion, we can find the image of the straight line $Y=M X+N$ in $\mathrm{Z}^{2}$ as follows:

$$
y=\frac{1}{p+2 M} x-\frac{2 N}{p+2 M} .
$$

The point $\left(\begin{array}{c}p v_{1}+2 v_{2}+2 \\ v_{1}+1\end{array}\right)$ is a mixed point in a concave boomerang of $C$. The vertical distance between this point and the line segment in (20) is:

$$
\begin{aligned}
\left(v_{1}+1\right)-\frac{p v_{1}+2 v_{2}+2-2 N}{p+2 M} & =\frac{2 M v_{1}+2 N-2 v_{2}-2+p+2 M}{p+2 M} \\
& \geq \frac{2-2+p+2 M}{p+2 M}=1 .
\end{aligned}
$$

Thus, the result in this case is also obvious by a contradiction with Theorem 3.2 .

Case (II), $\Leftarrow$. Conversely, we shall now prove the implication $\Leftarrow$ in case (II). Let $f(C) \in \mathrm{DSLS}_{8}$. As in Case $(\mathrm{I}), \Leftarrow$, we have the four possibilities which were mentioned in (14). First, we consider case 1. Thus, $C$ and $f(C)$ are dominant increasing and $C$ has dominant long boomerangs. Suppose that $C \notin \mathrm{DSLS}$. Therefore by Theorem 3.2, there is a mixed point $m$ of a boomerang $B$ such that the vertical distance at this point is at least one. We shall show that the maximal vertical distance in $C$ is attained at a mixed point $m=\left(m_{1}, m_{2}\right)^{\mathrm{T}}$ of a long boomerang, where the following boomerang is short. Suppose that this maximal vertical distance is attained between the mixed point $m$ and the line segment $[a, b]$ with equation $y=\alpha x+\beta$. We can see easily that we have the maximal vertical distance when $a$ is the starting point of a boomerang and $b$ is the endpoint of another boomerang. The slope of this line segment is

$$
\alpha=\frac{(r+s) p-2 s-r}{(r+s) p+r},
$$

where $r$ and $s$ are the number of long and short boomerangs, respectively. By a simple calculation, we find that the condition for the maximal vertical distance to be attained at a mixed point of the last long boomerang where the following boomerang is short, is

$$
\frac{p-2}{p} \leq \alpha \leq \frac{p-1}{p+1}
$$


We can see that the inequalities in (22) are correct by using (21). We can prove in the same way as for the case 3 in (14), that the maximal vertical distance is attained at the mixed point of a long boomerang where the following boomerang is short. As in the 8-connected case, for case 2 [4], we have maximal vertical distance at the mixed point of a long [long] boomerang where the previous boomerang is short [short]. With the same discussion as Case (I), $\Leftarrow$, we must show that the vertical distance at the image of the point $m^{\prime}=$ $\left(m_{1}+1, m_{2}\right)^{\mathrm{T}}$ in the grid $T$ is at least 1 . Let $m^{\prime \prime}$ be this image. Thus

$$
m^{\prime \prime}=\frac{1}{2}\left(\begin{array}{cc}
1 & -1 \\
2-p & p
\end{array}\right)\left(\begin{array}{c}
m_{1}+1 \\
m_{2}
\end{array}\right)=\frac{1}{2}\left(\begin{array}{c}
m_{1}-m_{2}+1 \\
(2-p)\left(m_{1}+1\right)+p m_{2}
\end{array}\right) .
$$

The point $m^{\prime \prime}$ is a vertex of a boomerang in $f(C)$. The image of a line segment $y=\alpha x+\beta$ in $T$ is

$$
Y=\frac{2-p+p \alpha}{1-\alpha} X+\frac{\beta}{1-\alpha} .
$$

Therefore, the vertical distance between line segment with equation (23) and the vertex $m^{\prime \prime}$ is

$$
\begin{aligned}
\frac{(2-p)\left(m_{1}+1\right)+p m_{2}}{2} & -\frac{2-p+p \alpha}{2(1-\alpha)}\left(m_{1}-m_{2}+1\right)-\frac{\beta}{1-\alpha} \\
& =\frac{2 m_{2}-2 \alpha m_{1}-2 \beta-2 \alpha}{2(1-\alpha)} \geq \frac{2-2 \alpha}{2(1-\alpha)}=1 .
\end{aligned}
$$

That is a contradiction. We can prove case 3 in (14) in the same way.

Let us now prove case 2 in (14). In this case, the maximal vertical distance is attained at the mixed point of a long boomerang where the previous boomerang is short. We consider the image of the point $m^{\prime}=\left(m_{1}-2, m_{2}-1\right)^{\mathrm{T}}$. The image of $m^{\prime}$ is the vertex of a convex boomerang in $f(C)$. We shall show that the vertical distance at this point is at least one. The image of the point $m^{\prime}$ can obtain as follows:

$$
m^{\prime \prime}=\left(\begin{array}{cc}
0 & 1 \\
\frac{1}{2} & -\frac{1}{2} p
\end{array}\right)\left(\begin{array}{l}
m_{1}-2 \\
m_{2}-1
\end{array}\right)=\left(\begin{array}{c}
m_{2}-1 \\
\frac{m_{1}+p-p m_{2}-2}{2}
\end{array}\right) .
$$

The image of a line segment $y=\alpha x+\beta$ in $T$ is

$$
Y=\frac{1-p \alpha}{2 \alpha} X-\frac{\beta}{2 \alpha} .
$$

Therefore, the vertical distance between line segment with equation (24) and the vertex $m^{\prime \prime}$ is

$$
\frac{1-p \alpha}{2 \alpha}\left(m_{2}-1\right)-\frac{\beta}{2 \alpha}-\frac{m_{1}+p-p m_{2}-2}{2}
$$




$$
=\frac{m_{2}-\alpha m_{1}-\beta+2 \alpha-1}{2 \alpha} \geq \frac{1+2 \alpha-1}{2 \alpha}=1 .
$$

Now Theorem 3.1 gives a contradiction. The case 4 in (14) can be proved in the same way.

Remark 4.10. The matrices $A$ in (12) have determinant \pm 1 so they have inverses with integer entries. The $2 \times 2$ matrices with determinant \pm 1 (called unimodular matrices) form a linear group $\mathrm{GL}(2, \mathrm{Z})$. Bruckstein [2] introduced such a transformation defined by $2 \times 2$ matrices with determinant \pm 1 . These matrices belong to $\mathrm{GL}(2, \mathrm{Z})$ and so have inverses in this group. He wrote that the image of all such transformations will provide chain codes of linearly separable dichotomies if and only if the transformed line induces a linearly separable dichotomy. Using this fact, he noted that all sequence transformations having this property yield chain codes for straight lines if and only if the original chain code is a digitized straight line. In the Khalimsky plane the matrices $A$ have determinant \pm 2 . Thus, they do not have such properties.

In the next theorem, we shall present another transformation to show the relation between $D S L S_{K h}$ and $D S L S_{8}$.

THEOREM 4.11. We define a function $g$ on a subset of the set $\{0,1\}^{\mathrm{N}}$ of sequences of zeros and ones and with values in the same set. For a chain code $C, g(C)$ is defined for dominant increasing or dominant constant sequences in the Khalimsky plane. We define $g(C)$ by replacing each pair of zeros by one zero. Then $C$ is the chain code of an element of DSLS $\mathrm{Kh}_{\text {if }}$ and only if $g(C) \in \mathrm{DSLS}_{8}$.

Proof. We define a transformation which gives us the chain codes of $g(C)$. To get the chain code of $g(C)$, we must replace 00 by 0 , and 1 by 1 . Thus, we define a grid $T$ same as the proof of Theorem 4.8, where

$$
V_{1}=\left(\begin{array}{c}
\frac{1}{2} \\
0
\end{array}\right) \quad \text { and } \quad V_{3}=\left(\begin{array}{c}
\frac{1}{2} \\
1
\end{array}\right) .
$$

We use the matrix $A=\left(\begin{array}{cc}\frac{1}{2} & \frac{1}{2} \\ 0 & 1\end{array}\right)$ for the equation in (11). We can come back from $T$ into $Z^{2}$ by using the matrix $A^{-1}=\left(\begin{array}{rr}2 & -1 \\ 0 & 1\end{array}\right)$ in (13).

We shall prove the implication $\Rightarrow$. Let $C \in \mathrm{DSLS}_{\mathrm{Kh}}$. Suppose that $g(C) \notin$ $\mathrm{DSLS}_{8}$. Thus, we can find a vertex $v$ such that the vertical distance between this point and the line segment with equation $Y=M X+N$ is at least one. The 
image of this line in $Z^{2}$ is

$$
y=\frac{M}{2-M} x+\frac{2 N}{2-M} .
$$

Let $v^{\prime}$ be the image of the point $v$ in $\mathrm{Z}^{2}$. Thus,

$$
v^{\prime}=\left(\begin{array}{cc}
2 & -1 \\
0 & 1
\end{array}\right)\left(\begin{array}{l}
v_{1} \\
v_{2}
\end{array}\right)=\left(\begin{array}{c}
2 v_{1}-v_{2} \\
v_{2}
\end{array}\right)
$$

It is clear that $v^{\prime}$ is a vertex of $C$. We shall show that the vertical distance between the mixed point $\left(2 v_{1}-v_{2}+1, v_{2}\right)^{\mathrm{T}}$ and the line segment with equation (25) is at least one. This vertical distance is

$$
\begin{aligned}
v_{2}-\frac{M}{2-M}\left(2 v_{1}-v_{2}+1\right) & -\frac{2 N}{2-M} \\
& =\frac{2\left(v_{2}-M v_{1}-N\right)-M}{2-M} \geq \frac{2-M}{2-M}=1 .
\end{aligned}
$$

This is a contradiction with Theorem 3.2.

We shall now prove the implication $\Leftarrow$. Let $g(C) \in \mathrm{DSLS}_{8}$. If $C \notin \mathrm{DSLS}$ Kh, then we can find a mixed point $m$ such that the vertical distance is at least one at this point. Suppose that the maximal vertical distance is attained between the mixed point $m$ and the line segment with equation $y=\alpha x+\beta$. We may assume that $m_{2} \geq \alpha m_{1}+\beta+1$. The proof for the case $m_{2} \leq \alpha m_{1}+\beta-1$ is similar. We consider the image of the point $m^{\prime}=\left(m_{1}-1, m_{2}\right)^{\mathrm{T}}$ in the grid $T$. Let this image be $m^{\prime \prime}$. Thus

$$
m^{\prime \prime}=\left(\begin{array}{cc}
\frac{1}{2} & \frac{1}{2} \\
0 & 1
\end{array}\right)\left(\begin{array}{c}
m_{1}-1 \\
m_{2}
\end{array}\right)=\left(\begin{array}{c}
\frac{1}{2}\left(m_{1}+m_{2}-1\right) \\
m_{2}
\end{array}\right) .
$$

The image of the line $y=\alpha x+\beta$ under $T$ is:

$$
Y=\frac{2 \alpha}{1+\alpha} X+\frac{\beta}{1+\alpha} .
$$

Therefore

$$
m_{2}-\frac{2 \alpha}{1+\alpha} \frac{m_{1}+m_{2}-1}{2}-\frac{\beta}{1+\alpha}=\frac{m_{2}-\alpha m_{1}-\beta+\alpha}{1+\alpha} \geq \frac{1+\alpha}{1+\alpha}=1 .
$$

Hence, we get a contradiction with Theorem 3.1. 


\section{Conclusion}

We studied digital lines in two different planes. One is the plane with connection by 8 -adjacency. The other plane which we studied here is the plane with topological structure. In this plane the connection is defined by the Khalimsky topology. We compare the Khalimsky plane and the 8-connected plane and to do so, we use a new concept, called boomerang (see Section 2). Using the vertices of boomerangs leads us to modify the chord property in both planes. These modification of chord property made the straightness easier to check.

We have investigated the properties of boomerangs which yield the necessary conditions for straightness. To find the sufficient conditions, we transform the chain codes of lines. By using this technique, we were be able to transfer questions on the Khalimsky plane to the 8-connected plane, and investigate straight lines using all the methods we have for the 8-connected plane.

AcKnOwledgments. I wish to thank Christer Kiselman for his comments and the time that he spent with me to complete this paper. I wish to thank Hanna Uscka-Wehlou for an interesting discussion on properties of digital straight line segments and finding some references on self-similarity. I also wish to thank Erik Melin for his comments on a preliminary version of this paper.

\section{REFERENCES}

1. Arnoux, Pierre, Berthé, Valérie, Fernique, Thomas, and Jamet, Damien, Functional stepped surfaces, flips, and generalized substitutions, Theoret. Comput. Sci. 380 (2007), 251-265.

2. Bruckstein, Alfred M., Self-similarity properties of digitized straight lines, pp. 1-20 in: Vision Geometry (Proc. Hoboken, NJ 1989), Contemp. Math. 119, Amer. Math. Soc., Providence, RI 1991.

3. Freeman, Herbert, Boundary encoding and processing, pp. 241-266 in: B. S. Lipkin and A. Rosenfeld (eds.), Picture Processing and Psychopictorics, Academic Press, New York 1970.

4. Hung, S. H. Y., Kasvand. T., On the chord property and its equivalences, pp. 116-119 in: Proceedings of the 7th International Conference on Pattern Recognition, Montreal 1984.

5. Jamet, Damien, and Toutant, Jean-Luc, On the connectedness of rational arithmetic discrete hyperplanes, pp. 223-234 in: A. Kuba, L. G. Nyúl, K. Palágyi (eds.), Discrete Geometry for Computer Imagery, Lecture Notes in Comput. Sci. 4245, Springer, Berlin 2006.

6. Khalimsky, Efim, Kopperman, Ralph, and Meyer, Paul R., Computer graphics and connected topologies on finite ordered sets, Topology Appl. 36 (1990), 1-17.

7. Kim, C. E., On cellular straight line segments, Computer Graphics and Image Processing 18 (1982), 369-381.

8. Kiselman, Christer O., Convex functions on discrete sets, pp. 443-457 in: R. Klette and J. Žunić (eds.), Combinatorial Image Analysis, Lecture Notes in Comput. Sci. 3322, Springer, Berlin 2004.

9. Kiselman, Christer O., Digital Geometry and Mathematical Morphology, Lecture notes, Uppsala University 2004. 
10. Klette, Reinhard, and Rosenfeld, Azriel, Digital Geometry. Geometric Methods for Digital Picture Analysis, Elsevier, Amsterdam 2004.

11. Klette, Reinhard, and Rosenfeld, Azriel, Digital straightness—a review, Discrete Appl. Math. 139 (2004), 197-230.

12. Maloň, Stanislaw, and Freeman, Herbert, On the encoding of arbitrary geometric configurations, IRE Trans. EC-10 (1961), 260-268.

13. Melin, Erik, Digital straight lines in the Khalimsky plane, Math. Scand. 96 (2005), 49-64.

14. Melin, Erik, Digital Geometry and Khalimsky Spaces, Ph.D. Thesis, Uppsala Dissertations in Mathematics 54, Uppsala University, Uppsala 2008.

15. Reveillès, Jean-Pierre, Géométrie discrète, calcul en nombres entiers et algorithmique, Ph.D. Thesis, Université Louis Pasteur, Strasbourg 1991.

16. Rosenfeld, Azriel, Digital straight line segments, IEEE Trans. Computers. C-23 (1974), 1264 1269.

17. Smeulders, A. W. M, and Dorst, L., Decomposition of discrete curves into piecewise straight segments in linear time, pp. 169-195 in: Vision Geometry (Proc. Hoboken, NJ 1989), Contemp. Math. 119, Amer. Math. Soc., Providence, RI 1991.

18. Uscka-Wehlou, Hanna, Digital lines with irrational slopes, Theoret. Comput. Sci. 377 (2007), 157-169.

\author{
DEPARTMENT OF MATHEMATICS \\ STOCKHOLM UNIVERSITY \\ STOCKHOLM \\ SWEDEN \\ E-mail: shiva@math.su.se
}

